\title{
Estrategias de mejora del Prácticum del Grado en Sociología a través de un modelo CAME
}

\author{
Román-Masedo, Laura'; Otero-Enríquez, Raimundo²; Rodríguez-Teijeiro, Ariadna ${ }^{3}$ \\ ${ }^{1}$ Profesora Titular de Universidad, Facultad de Sociología, orcid.org/0000-0001-7383-9980 \\ ${ }^{2}$ Profesor Ayudante Doctor, Facultad de Sociología, orcid.org/0000-0002-8074-3765 \\ ${ }^{3}$ Profesora Ayudante Doctora, Facultad de Sociología, orcid.org/0000-0001-7582-650X
}

\section{RESUMEN}

En el marco del Grado en Sociología de la Universidade da Coruña, las prácticas curriculares -0 Prácticum- se disponen en el plan de estudios como una asignatura de carácter obligatorio, con una importante carga lectiva de 12 créditos ECTS. En el presente texto se pretende profundizar tanto en aspectos que suponen una problemática para esta asignatura, como en los que plantean una posibilidad de mejora. Con este objetivo, se ha elaborado una matriz de estrategias y medidas (análisis CAME) encaminada a corregir, afrontar, mantener y explotar las debilidades, amenazas, fortalezas y oportunidades del Prácticum que han sido detectadas durante los últimos cursos académicos, gracias a un sistema de evaluación basado en la aplicación de técnicas cuantitativas y cualitativas de investigación.

PALABRAS CLAVE: Estrategias, CAME, Prácticum, gestión, aprendizaje. 


\section{CITA RECOMENDADA:}

Román-Masedo, Laura; Otero-Enríquez, Raimundo; Rodríguez-Teijeiro, Ariadna (2020): Estrategias de mejora del Prácticum del Grado en Sociología a través de un modelo CAME. En De la Torre Fernández, E. (ed.) (2020). Contextos universitarios transformadores: Boas prácticas no marco dos GID. IV Xornadas de Innovación Docente. Cufie. Universidade da Coruña. A Coruña (págs. 435-446).

DOI capítulo: https://doi.org/10.17979/spudc. 9788497497756.435

DOl libro: https://doi.org/10.17979/spudc. 9788497497756

\section{ABSTRACT}

Within the framework of the Degree in Sociology of the University of A Coruña, curricular internships -or Practicum- are arranged in the curriculum as a compulsory subject, with an important teaching load of 12 ECTS credits. This text aims to deepen into aspects that are problematic for this subject, as well as those that pose a possibility of improvement. With this objective, a matrix of strategies and measures (CAME analysis) has been designed to correct, adapt, maintain and explore the weaknesses, threats, strengths and opportunities of the Practicum that have been detected during the last academic years, due to a system of evaluation based on the application of quantitative and qualitative research techniques.

KEY WORDS: Strategies, CAME, practicum, management, learning. 


\section{INTRODUCCIÓN}

En el marco del Espacio Europeo de Educación Superior (EEES), la utilización de nuevos métodos de evaluación es uno de los objetivos prioritarios en materia de innovación educativa. En este contexto, la aplicación de técnicas prospectivas de análisis se vislumbra como una herramienta apropiada de anticipación y toma de decisiones que puede resultar muy útil de cara a su aplicación en la gestión y mejora en este ámbito educativo. Dicha perspectiva nos permite adentrarnos en el diagnóstico de los aspectos de naturaleza interna y externa del Prácticum curricular del Grado en Sociología de la Universidade da Coruña (UDC).

En este Grado, las prácticas curriculares se disponen en el plan de estudios como una asignatura de carácter obligatorio, con una importante carga lectiva de 12 créditos ECTS, y se desarrollan en el segundo cuatrimestre del cuarto y último curso junto con la realización del Trabajo de Fin de Grado (TFG), de temática abierta, y también con una carga idéntica de 12 créditos ECTS. En concreto, dichas prácticas obligan a nuestro alumnado a vivir una experiencia profesional-formativa en centros de diversa naturaleza (normalmente consultoras de estudios de mercado o de recursos humanos, administraciones públicas, instituciones del Tercer Sector, etc.), durante un total de 275 horas.

Partiendo nuestro análisis de un sistema de evaluación ad hoc puesto en marcha desde el curso 2014-15, basado en la aplicación de técnicas cuantitativas y cualitativas de investigación, las evidencias obtenidas nos han permitido alcanzar una visión profunda de la realidad de este Prácticum curricular, que se ha tratado de plasmar en su mejor gestión (es preciso señalar que los autores de este texto han sido los coordinadores de la materia en los últimos años).

En primer lugar, en el curso 2017-18 comienza su andadura el Grupo de Innovación Educativa Prácticum (GIE Prácticum), concebido como un marco colaborativo para mejorar la evaluación y gestión de las prácticas curriculares de las siguientes titulaciones pertenecientes a las Facultades de Sociología y Ciencias de la Comunicación de la UDC: Grado en Comunicación, Grado en Sociología, Máster Universitario en Sociología Aplicada y Máster Universitario en 
Políticas Sociales. Precisamente, para ser presentado y discutido en el contexto del GIE Prácticum, se pone en marcha una primera fase de análisis consistente en la detección de debilidades, amenazas, fortalezas y oportunidades (análisis DAFO) de las prácticas curriculares del Grado en Sociología, a partir de los resultados obtenidos del sistema de evaluación antes referido. La aplicación de dicha técnica prospectiva nos permitió obtener un diagnóstico exhaustivo del estado del Prácticum en el que se delimitan dos escenarios de futuro que será necesario discutir y contrastar.

En una segunda fase de análisis, se elabora una matriz de estrategias y medidas (análisis CAME) encaminadas a corregir, afrontar, mantener y explotar los aspectos identificados en el DAFO. De esta manera, se da un paso más a la hora establecer unas medidas claras de actuación de un Prácticum que se encuentra ante diversos retos a acometer para su mejora, y ante una situación compleja en el corto plazo (principalmente, debido al probable desarrollo reglamentario de un Real Decreto Ley que obligaría a dar de baja y de alta en la Seguridad Social al alumnado en prácticas).

El objetivo de este texto es explicar con detalle estas fases de análisis y reflexión, así como finalmente exponer las medidas de mejora detectadas mediante un análisis CAME, las cuales nos pueden permitir ubicar a la asignatura en un escenario futuro, pensando en el rendimiento académico óptimo del alumnado.

\section{DESCRIPCIÓN DE LA EXPERIENCIA}

Es indudable que las técnicas prospectivas de análisis (DAFO 0 CAME) son herramientas apropiadas de cara a su aplicación en la gestión y mejora en el ámbito educativo, especialmente para poner en marcha nuevas experiencias de evaluación estratégica en los centros educativos (Moral, 2010; Lumillo et al., 2013; Ayala et al., 2017). Partiendo de esta premisa, la utilidad de dichas técnicas sería completamente ineficiente sin recabar evidencias previas extraídas de un sistema de evaluación sustentando en una triangulación metodológica 
(Rodríguez, 2005) o secuencia inter-métodos. Este, con más detalle, se compone de dos elementos principalesi:

- Una completa encuesta de evaluación que el alumnado cubre tras la finalización de las prácticas curriculares (y que se empieza a cubrir en el curso 2015-16 hasta el presente).

- La realización periódica de grupos de discusión formados por estudiantes egresados de diferentes promociones del Grado (el último se celebró en el curso 2018-19).

De manera transversal, tanto los cuestionarios como el guion abierto de los grupos de discusión observan las mismas dimensiones de evaluación, que consisten en la valoración por parte del alumnado de: a) la gestión del Prácticum; b) el entorno profesional de los centros de prácticas; c) la utilidad de los conocimientos adquiridos en el Grado; d) las motivaciones/emociones individuales presentes en las prácticas; y e) las competencias desarrolladas y resultados de aprendizaje alcanzados.

Por tanto, teniendo sobre la mesa múltiples evidencias cuantitativas y cualitativasii, los coordinadores de la asignatura generan un primer análisis DAFO (Debilidades, Amenazas, Fortalezas y Oportunidades), que se expone a la totalidad del GIE Prácticum. Este DAFO tiene la particularidad de que sirve para trazar escenarios probables en relación al futuro del Prácticum (Vergara, 2010). Dicha particularidad es necesaria habida cuenta de que la institución universitaria, desde la implantación del EESS, continúa inmersa en un proceso constante y vertiginoso de cambio organizativo, especialmente negativo para los sistemas de prácticas curriculares de naturaleza poliédrica (Zabalza, 2011: 35).

Desagregando las dimensiones del DAFO (Rodríguez-Teijeiro et al., 2019a), en el marco de las debilidades sobresale la falta de infraestructuras administrativas de apoyo a la gestión, lo cual dificulta las labores de coordinación de esta asignatura (por ejemplo, a la hora de gestionar y actualizar los convenios a partir de los cuales se oficializa la incorporación de los centros al sistema de prácticas). También resulta particularmente problemática la convivencia en un 
mismo cuatrimestre del Prácticum con la realización del TFG, vista como incompatible por una parte importante de nuestro estudiantado (Tabla 1).

\begin{tabular}{c|c}
\hline FACTORES INTERNOS & FACTORES EXTERNOS \\
\hline DEBILIDADES (-) & AMENAZAS (-) \\
\hline $\begin{array}{c}\text { (D1) Ausencia de infraestructuras burocráticas } \\
\text { (D2) Escasa relación institucional con algunos centros } \\
\text { de prácticas }\end{array}$ & (A1) Complejidad normativa creciente (Real Decreto \\
(D3) Difícil coexistencia entre las prácticas y los TFG & (A3) Falta de centros de prácticas en el sector \\
\end{tabular}

\begin{tabular}{|c|c|}
\hline \multicolumn{2}{|c|}{ (- -) Escenario probable: "PRÁCTICUM REGRESIVO" } \\
\hline FORTALEZAS (+) & OPORTUNIDADES (+) \\
\hline (F1) Nivel de institucionalización óptimo & $\begin{array}{l}\text { (01) Papel óptimo como plataforma de inserción } \\
\text { laboral }\end{array}$ \\
\hline $\begin{array}{c}\text { (F2) Número abultado de convenios de prácticas } \\
\text { vigentes }\end{array}$ & $\begin{array}{l}\text { (02) Valía como proceso formativo y de aprendizaje } \\
\text { personal }\end{array}$ \\
\hline (F3) Sistema de evaluación inter-métodos & (03) "Recorrido futuro" como asignatura \\
\hline (F4) Medidas de mejora ya implementadas & $\begin{array}{c}\text { (04) Búsqueda de puntos comunes entre las } \\
\text { prácticas y los TFG }\end{array}$ \\
\hline +) Escenario probabl & ZÁCTICUM CURRICULAR" \\
\hline
\end{tabular}

Tabla 1. Modelo DAFO y proyección de escenarios. Fuente: elaboración propia

Respecto a las amenazas, la más clara es la referida al probable desarrollo reglamentario de la Disposición Adicional Quinta del Real Decreto Ley 28/2018 (BOE de 29 de diciembre), según la cual habría que dar de alta en el Régimen General de la Seguridad Social a los estudiantes de las prácticas curriculares. La falta de una información concreta sobre el futuro de la aplicación de esta medida, está generando una enorme incertidumbre sobre la organización futura -y sostenibilidad- de los Prácticum en el sistema universitario españoliii.

Otra amenaza importante es la de no estar consiguiendo convenios suficientes con centros de prácticas del sector industrial o secundario en los cuáles, quizás, no existe un conocimiento adecuado sobre las potencialidades laborales de la Sociología. Más allá de esta cuestión, este sector puede ofrecer oportunidades laborales a nuestros egresados (en la gestión de recursos humanos, big data, análisis de calidad, etc.) muy prometedoras. 
Por el contrario, en el contexto de las oportunidades del DAFO (Tabla 1), se valoran dos aspectos clave: a) las prácticas del Grado en Sociología se están consolidando como una plataforma de inserción laboral óptima ${ }^{\text {iv }}$ y b) tal y como muestran las encuestas realizadas en Ios últimos cursos, el Prácticum está facilitando la asimilación por parte del alumnado de valiosas competencias y resultados de aprendizaje (Rodríguez-Teijeiro et al., 2019b: 14-16). En el cuadrante de las fortalezas, por ejemplo, no cabe duda de que la asignatura cuenta con un alto grado de institucionalización en el Centro, se sigue actualizando y perfeccionando el sistema de evaluación inter-métodos curso a curso, y han sido varias las medidas de mejora implementadas (por ejemplo, la confección de un manual-guía de la asignatura, o la celebración desde hace cuatro cursos de una jornada de encuentro entre el alumnado y los centros de prácticas).

A continuación, la reflexión que realizan los coordinadores del Prácticum sobre el DAFO resultante, traza dos escenarios futuros contrapuestos (Tabla 1):

- 1\% El escenario de un "Prácticum regresivo" en el que las debilidades y amenazas aumentan su importancia. En este contexto, la asignatura no contaría con una infraestructura burocrática necesaria para su adecuada gestión, y no sería capaz de hacer frente con eficacia a los grandes retos burocráticos, jurídicos y económicos que se derivan de la aplicación del Real Decreto Ley 28/2018. En consecuencia, el número de convenios con nuevos centros de prácticas se reduciría drásticamente y tanto la satisfacción del alumnado, como la de los centros involucrados disminuiría. También sería probable que la asignatura se convirtiese en una materia "optativa", con un número de créditos ECTS menor, ante la imposibilidad de asignar un centro de prácticas a la totalidad del alumnado matriculadov .

- $2 \%$ El escenario de un "Prácticum puramente curricular", que seguiría siendo una materia obligatoria de la Titulación. En este contexto, la asignatura habría solventado los principales problemas que se pueden originar debido al desarrollo del Real Decreto Ley mencionado. Por tanto, seguiría constituyendo una base formativa óptima para el 
estudiantado a la hora de asumir competencias profesionales clave. También contaría con una infraestructura burocrática sólida de apoyo, y con un grado alto de reconocimiento institucional en tanto en cuanto enlace indispensable entre la Universidad y el mercado laboral. Asimismo, este Prácticum conseguiría excelentes cotas de satisfacción profesional y personal del estudiantado.

A continuación, trazados ambos escenarios, se activa una segunda fase de prospección sustentada en un análisis CAME (Tabla 2). La matriz resultante, una evolución lógica del DAFO previo, constituye un instrumento que nos permite corregir las debilidades y mantener las fortalezas detectadas, así como afrontar las amenazas y explotar las oportunidades a partir del diseño de una serie de propuestas específicas de actuación (De León et. al., 2011: 74). Éstas se exponen en el siguiente apartado de resultados y conclusiones.

\begin{tabular}{c|c}
\hline FACTORES INTERNOS & FACTORES EXTERNOS \\
\hline $\begin{array}{c}\text { CORREGIR LAS DEBILIDADES } \\
\text { dejen de existir, o de afectar negativamente }\end{array}$ & $\begin{array}{c}\text { Evitar que las amenazas se conviertan en } \\
\text { debilidades, tomando medidas para que éstas } \\
\text { desaparezcan, o para reducir su impacto }\end{array}$ \\
\hline MANTENER LAS FORTALEZAS & EXPLOTAR LAS OPORTUNIDADES \\
\hline $\begin{array}{c}\text { Tomar medidas para mantener los puntos fuertes y sacar } \\
\text { partido a los aciertos }\end{array}$ & $\begin{array}{c}\text { Establecer estrategias y acciones encaminadas a } \\
\text { convertir las oportunidades en futuras fortalezas }\end{array}$ \\
\hline
\end{tabular}

Tabla 2. Modelo CAME. Fuente: elaboración propia

\section{RESULTADOS Y CONCLUSIONES}

En concreto, entre los resultados más relevantes que arroja la aplicación del modelo CAME se encuentran las principales (que no únicas) vías de acción para la mejora del Prácticum en el corto plazo:

- 10/ Corregir las deficiencias relativas a la gestión burocrática de la asignatura (D1). En este sentido, y gracias al apoyo del equipo decanal del Centro, ha resultado muy importante que el personal administrativo colabore más activamente en la gestión de 
la sistematización de la documentación ad hoc generada por las prácticas (por ejemplo, certificados que acreditan la realización de las prácticas para cada alumno), y en la actualización de bases de datos (por ejemplo, de convenios firmados con los centros). Esto ha permitido al equipo coordinador de la asignatura destinar más tiempo a otras tareas de organización docente y tutorial de carácter estratégico. Por supuesto, en los próximos cursos será fundamental seguir manteniendo esta colaboración.

- $2^{\circ} /$ Corregir la difícil coexistencia del Prácticum y el TFG en el mismo cuatrimestre del cuarto curso del Grado (D3). Para que esto ocurriera, habría que cambiar el plan de estudios del actual Grado en Sociología de la UDC. Más allá de lo deseable o no de la medida, esta iniciativa queda lógicamente fuera del alcance de los coordinadores del Prácticum. Sin embargo, se está evaluando la posibilidad de que las prácticas curriculares desarrolladas en centros pertenecientes al Tercer Sector se enfoquen como actividades de Aprendizaje-Servicio $(A p S)^{\text {vi }}$. Tales actividades podrían plasmarse (a modo de diagnóstico de una realidad social concreta, por ejemplo) en la elaboración de un TFG. De esta forma, los alumnos que hubiesen sido seleccionados para este tipo de centros podrían realizar sus respectivos TFG al mismo tiempo que sus prácticas.

- $3^{\circ} /$ Afrontar los principales retos derivados fundamentalmente del desarrollo del Real Decreto Ley 28/2018, según el cual los estudiantes en prácticas habrán de ser dados de alta en el régimen de la Seguridad Social (A1). Son muchas las incógnitas que están todavía por despejar en caso de que el desarrollo íntegro de esta norma se haga realidad (por ejemplo, ¿quién se va a hacer cargo del coste del alta en la Seguridad Social?). Lo que sí sería probable es que, por los costes económicos derivados, la falta de cultura organizativa y diversas problemáticas jurídico-laborales, una gran parte de los centros de prácticas se desvincularía del sistema de prácticas del Grado en Sociología. La incorrecta aplicación de esta medida, al menos durante los primeros 
años de la aplicación de la nueva norma, puede suponer que el Prácticum se escore hacia los parámetros del "escenario regresivo" antes descrito. Desgraciadamente, esta es una acción externa sobre la que el equipo coordinador no tiene ningún margen de maniobra (tan solo, llegado el caso, el de comunicar con claridad las consecuencias que implicaría esta nueva normativa a los centros de prácticas).

- 4\% Afrontar la infrarrepresentación de centros de prácticas pertenecientes al sector secundario, o a nichos laborales en los cuales la Sociología no tiene un alto grado de reconocimiento profesional o institucional (A3). Para ello, se presentará en los próximos meses un "Protocolo de acogida del alumno" en el que se informará a los centros de prácticas nuevos o de potencial interés en qué consiste el Prácticum, así como los pasos a seguir para una efectiva integración formativa y profesional del estudiante en los mismos. Otras finalidades del "Protocolo" también serán explicar cuál es el "oficio" del sociólogo y las competencias adquiridas en el Grado por parte de nuestro alumnado, así como poner de relieve los aspectos que más valora éste en sus prácticas.

- 5\% Mantener las líneas de mejora que hasta el momento han permitido potenciar las principales fortalezas del Prácticum (F3). Esto conlleva, principalmente, seguir mejorando y aplicando el sistema de evaluación inter-métodos de cara a la detección de nuevas variables (sean competencias, resultados de aprendizaje 0 motivaciones individuales) que conlleven una mayor satisfacción por parte de los centros de prácticas y del alumnado.

- 6\% Explotar aquellos posibles espacios de mejora y oportunidades (01). Por ejemplo, resulta fundamental seguir visibilizando, y comunicando tanto al alumnado como al personal docente e investigador del Centro, hasta qué punto esta materia es una plataforma importante de inserción laboral que debe seguir teniendo un peso relevante en el plan de estudios (vigente 0 futuro). 
Por último, apuntar que estas acciones de mejora serán puestas en común en los próximos meses en el marco colaborativo del GIE Prácticum. No sólo se trata de acotar estas estrategias evaluadoras, prospectivas y planificadoras en el ámbito del Grado en Sociología, sino de contrastarlas y, llegado el caso, aplicarlas en los restantes sistemas de prácticas de las Titulaciones integrantes de dicho Grupo.

\section{REFERENCIAS}

Ayala, I.; Villa-González, E.; Barraco-Ruiz, Y. \& Chillón, P. (2017). Innovación educativa para el fomento del desplazamiento activo al instituto: análisis de viabilidad y propuesta de intervención. Trances, 9(3), pp. 493-424.

De León, C.; González, I.; López, I. \& López, A.B. (2011). Repertorio de estrategias de evaluación de competencias educativas en el aula universitaria. En EVALfor (Ed.), Evaluar para aprender en la universidad: experiencias innovadoras en la sistematización de la evaluación (pp. 68-77). Madrid: Bubok Publishing.

Lumillo, I.; Sánchez, B.; Garzón, M.; Gallardo C. \& Salvadó, M.C. (2013). Análisis CAME-DAFO del primer semestre como Residentes de Enfermería Familiar y Comunitaria. Metas de Enfermería, 16(5), pp. 62-66.

Moral-López, A.; Arrabal-Gómez, I. \& González-López, I. (2010). Nuevas experiencias de evaluación estratégica en los centros educativos. La aplicación de una matriz DAFO en el centro de educación infantil y primaria "Mediterráneo" de Córdoba. Estudios sobre educación, 18, pp. 165-200.

Rodríguez, 0. (2005). La triangulación como estrategia de investigación en Ciencias Sociales. Revista de Investigación en Gestión de la Innovación y Tecnología, 31, pp. 1-10.

Rodríguez-Teijeiro, A.; Román-Masedo, L. \& Otero-Enríquez, R. (2019a). El futuro del Prácticum: un análisis prospectivo (en prensa). Comunicación presentada en el Congreso Internacional de Docencia Universitaria CINDU 2019. Futuro de la docencia en la universidad. Vigo, Junio de 2019. 
Rodríguez-Teijeiro, A.; Otero-Enríquez, R. \& Román-Masedo, L. (2019b). Prácticas curriculares y Sociología en la Universidade da Coruña: de las competencias a las experiencias profesionales y personales. Revista Practicum, 4(2), pp. 4-23.

Vergara, J.; Fontalvo, T.J. \& Maza F. (2010). La planeación por escenarios: revisión de conceptos y propuestas metodológicas. Prospectiva, 8(2), pp. 21-29.

Zabalza, M. (2011). El Practicum en la formación universitaria: estado de la cuestión. Revista de Educación, 354, pp. 21-43.

' El sistema de evaluación también se sustenta en la realización de una segunda encuesta on-line a alumnos egresados (que llevan al menos cinco años titulados), que se dirige a medir la utilidad pasada de las prácticas como herramienta para la inserción en el mercado laboral. El sistema también se compone de una tercera encuesta destinada a analizar el grado de satisfacción de los tutores profesionales de cada centro de prácticas con el desempeño profesional del alumnado.

ii Véase un compendio crítico del análisis de los datos -cuantitativos y cualitativos- conseguidos por el sistema de evaluación, en Rodríguez-Teijeiro et al. (2019b).

iii Como así ha reconocido la CRUE en diferentes notas de prensa, tras la aprobación del Real Decreto Ley. Véase el siguiente enlace (consultado el 13 de enero de 2020): http://www.crue.org/Documentos\%20compartidos/Comunicados/2019.01.04.\%20NdP\%20Crue\%20Pr a\%CC\% 81 cticas\%20externas.pdf

${ }^{i v}$ A falta de analizar los últimos datos disponibles, un $16 \%$ del alumnado egresado de la Titulación, entre los cursos 2015-16 y 2017-18 y una vez finalizadas sus prácticas curriculares, tuvo la posibilidad de firmar un contrato laboral. En este sentido, más de un $48 \%$ manifestó la posibilidad, en un futuro, de continuar vinculado laboralmente con su centro de prácticas.

${ }^{\vee}$ En los últimos tres cursos académicos, el número de alumnos matriculados se ha estabilizado entre los 45 y 55. Es previsible que esta cifra aumente en los próximos años.

vi Véase el siguiente enlace (consultado el 13 de enero de 2020) para obtener más información sobre este tipo de aprendizaje que integra un servicio social a una comunidad: https://www.udc.es/es/cufie/INNOVACION/aps/ 